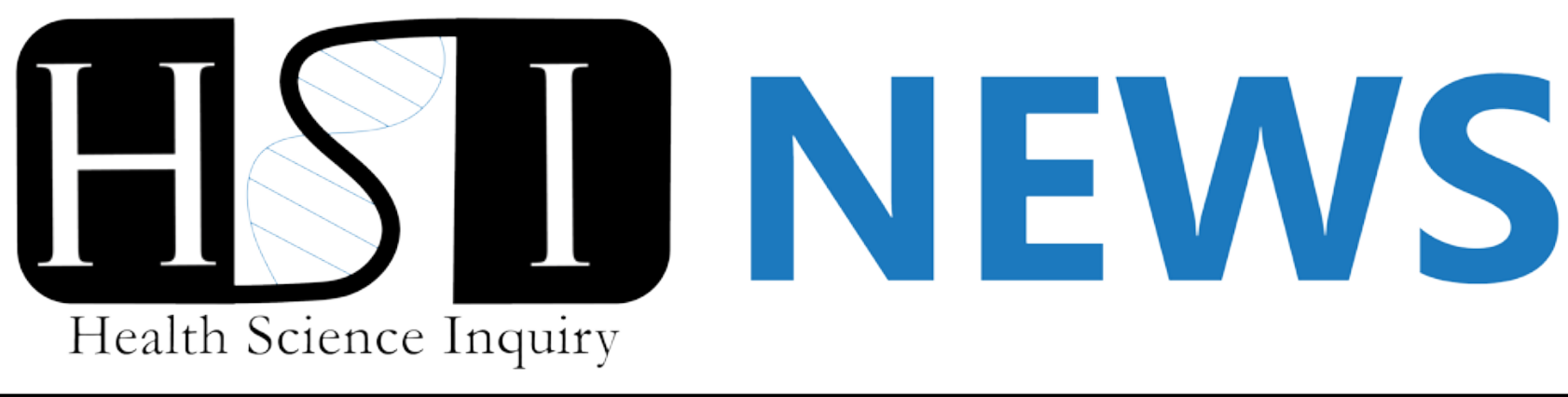

\title{
An Unfortunate Interaction Between Anti-Psychotic Medication and Obesity
}

\author{
- Logan Townsend
}

$S$ econd generation antipsychotic (SGAs) drugs, like olanzapine, are the current standard-of-care for the treatment of schizophrenia because they are more tolerable than earlier drugs. In recent years, SGAs have been increasingly used for managing a range of common off-label conditions such as anxiety, attention deficit, bipolar, and sleep disorders. Partly because of this off-label use, the number of SGA prescriptions in Canada more than doubled to $>7$ million annually between 2005-2012. Unfortunately, SGAs have serious metabolic side effects, including weight gain and type 2 diabetes, affecting up to $70 \%$ of individuals taking these drugs [1].

Initially, it was believed that the metabolic side effects associated with SGAs were secondary to weight gain, but it is clear that SGAs have direct diabetogenic effects. In both rodents and humans, SGAs cause high blood sugar within minutes [2]. Acute SGA-induced hyperglycemia does not dissipate even after months of continuous use [2], and is linked to adverse effects on the brain, liver, muscle, and fat tissue [3].

Regardless of SGA use, obesity is more prevalent in individuals with schizophrenia compared to the general population. This raises the important question of whether the metabolic dysfunction associated with obesity could alter the acute responses to SGAs. Indeed, olanzapine's side effects mimick many of the metabolic disturbances that characterize obesity, including hyperglycemia, insulin resistance, and glucose intolerance. Moreover, Dr. David Wright at the University of Guelph reasoned that "initial studies [testing the responses to olanzapine] have all used lean, healthy rodents. As individuals taking antipsychotics are often obese and display perturbations in glucose metabolism prior to the onset of taking medication we thought that this could impact the acute metabolic side effects of antipsychotics."

With this in mind, Dr. Wright and I fed $~ 30$ mice a high-fat diet to make them obese. After a month, we confirmed that obese mice displayed the classic characteristics of obesity, like insulin resistance. We then performed a series of experiments comparing the metabolic responses to olanzapine in these obese mice versus their lean counterparts. Overall, Dr. Wright said that "mice fed the high fat diet developed more severe hyperglycaemia with olanzapine treatment and this was associated with a greater degree of insulin resistance and increases in markers of liver glucose production." From Dr. Wright's perspective, these findings have direct implications for humans: "acute side effects of olanzapine and other antipsychotic drugs are even more severe than initially envisioned, as many of the individuals given this drugs are obese and glucose intolerant prior to treatment."

These findings have relevance to not only those with schizophrenia, but the entire patient population who are prescribed SGAs for various conditions. The current obesity epidemic means that those taking SGAs are very likely experiencing more severe side effects than they realize [3]. Moreover, SGAs, especially olanzapine, rapidly cause weight gain meaning that side effects may get progressively worse with chronic use. This cycle may partly explain why SGA use shortens the timeframe of weight gain to diabetes from decades to months [1].

Dr. Wright's group is now working to identify potential ways to alleviate some of Olanzapine's adverse side-effects, particularly glucose regulation. Multiple groups have previously tried using diabetic medications, given that SGAs side effects are similar to many diabetic symptoms, but with only partial success. For example, metformin, the most commonly prescribed anti-diabetic drug, was only partially able to prevent SGA-induced hyperglycemia [2]. We suspect that the moderate success of metformin may be due to the fact that it only functions in the liver, whereas our team's research has shown that olanzapine functions in both the liver and skeletal muscle [3]. Therefore, Dr. Wright believes a more 
whole-body intervention will be required. For instance, they very recently published a report showing that exhaustive exercise can completely block the acute olanzapine-induced hyperglycemia [4]. However, exhaustive exercise is a very impractical strategy in humans, especially since it needs to be performed immediately before every olanzapine dose (daily!). For this reason, they are planning to experiment with "exercise mimetics," essentially drugs that attempt to mimic the benefits of exercise, as a novel way to combat the unwanted side effects of SGAs.

Taken together, there appears to be an unfortunate interaction between SGAs, including olanzapine, and obesity. With the increasing prescription of SGAs and epidemic of obesity, these findings have far-reaching implications. It will be important for future work to consider their treatment groups, likely including obese animal models and human participants.

\section{References}

[1] Rojo, L.E., Gaspar, P.A., Silva, H., Risco, L., Arena, P., Cubillos-Robles, K., Jara, B. (2015) Metabolic syndrome and obesity among users of second generation antipsychotics: A global challenge for modern psychopharmacology. Pharmacological research, 101, 74-85.

[2] Boyda, H.N., Tse, L., Procyshyn, R.M., Honer, W.G., Barr, A.M. (2010) Preclinical models of antipsychotic druginduced metabolic side effects. Trends in Pharmacological Sciences, 31 (10), 484-497.

[3] Townsend, L.K., Peppler, W.T., Bush, N.D., Wright, D.C. (2018) Obesity exacerbates the acute metabolic side effects of olanzapine. Psychoneuroendocrinology, 88, 121-128.

[4] Castellani, L.N., Peppler, W.T., Miotto, P.M., Bush, N., Wright, D.C. (2018) Exercise Protects Against OlanzapineInduced Hyperglycemia in Male C57BL/6J Mice. Scientific reports, 8 (1), 772.

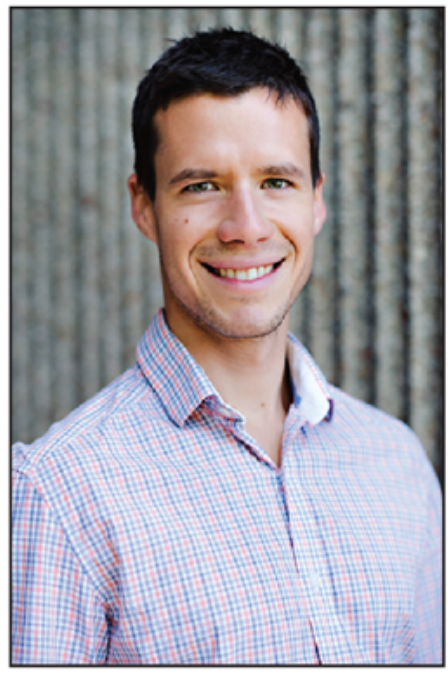

Logan is a PhD candidate at the University of Guelph. His research looks at metabolic contributors to the development of diabetes. Specifically he focuses on liver and adipose tissue and the effects of interleukin-6. In his spare time he enjoys rock climbing and mountain biking. 